\title{
Lifestyle Habits and Wellbeing Among Physicians in Pakistan: A Cross-Sectional Study
}

\author{
Anum S. Siddiqui ${ }^{1}$, Zubair Siddiqui ${ }^{2}$, Ramsha Khulsai ${ }^{1}$, Masood Jawaid ${ }^{3}$ \\ 1. Medical Affairs, PharmEvo Private Limited, Karachi, PAK 2. Marketing, PharmEvo Private Limited, Karachi, PAK 3. \\ Surgery, Darul Sehat Hospital, Karachi, PAK
}

Corresponding author: Anum S. Siddiqui, anumsohailsiddiqui@gmail.com

\section{Abstract \\ Introduction}

Physicians' attitude towards a healthy lifestyle is important as it determines their extent of acceptance of these habits leading to overall well-being. Physicians with healthy lifestyle habits are more confident in advocating the same to their patients and their patients are keener in adopting lifestyle modifications. This study aimed to evaluate the lifestyle habits, wellbeing, and mental health of physicians in Pakistan.

\section{Methods}

A multi-center, cross-sectional survey was conducted with physicians across Pakistan from August to October 2020. A total of 1406 participants were gathered by the non-probability convenient sampling technique. Data were collected physically from the participants. A semi-structured, self-administered questionnaire comprising socio-demographic information, lifestyle habits, mental well-being, and wellbeing, in general, was filled. Mental wellbeing was assessed using the Warwick Edinburg Mental Wellbeing Scale (WEMWBS) comprising 14-positive items scored on a 1-5 point Likert scale where 1 corresponds to "none of the time" and 5 corresponds to "all of the time." The score ranges from a minimum of 14 to a maximum of 70 points. Higher scores are associated with higher levels of mental wellbeing. Data were stored and analyzed using IBM-SPSS v.23 (IBM Corp., Armonk, NY).

\section{Results}

In this survey, there were 1284 (91.3\%) males and 122 (8.7\%) females with a mean age of $44.09 \pm 11.18$ years. More than half ( $\mathrm{n}=768$; 53.0\%) of the physicians reported their general health as "good," 1045 (73\%) were satisfied with their work-life balance, 206 (14\%) had seen a physician for their health in the last six months, and $358(25 \%)$ never had a routine medical checkup. When WEMWBS was applied, participants $\leqslant 40$ years scored significantly higher than the older age group $(\mathrm{p}<0.01)$. Male physicians also scored significantly higher on WEMWBS $(52.35 \pm 8.78)$ as compared to their female counterparts $(\mathrm{p}<0.01)$. WEMWBS scores also varied significantly across various levels of expertise - with consultants scoring the highest $(52.67 \pm 9.02)$ and others scoring the lowest $(48.63 \pm 8.58 ; \mathrm{p}=0.02$ ). Physicians practicing in the public hospitals only ( $53.05 \pm$ $9.02)$, scored higher on WEMWBS as compared to those in the private hospitals $(51.28 \pm 8.12)$ as well as those practicing in private clinics only $(49.57 \pm 8.82 ; \mathrm{p}<0.01)$. Physicians who perceived their health as excellent scored highest on WEMWBS $(53.55 \pm 9.31)$, than those who considered their health good $(51.68 \pm 8.40)$, poor $(50.71 \pm 9.27)$, or fair $(48.70 \pm 8.15 ; \mathrm{p}<0.01)$. The correlation analysis showed a significant negative correlation of WEMWBS scores with health in general ( $2.5 \%$ variation) and age $(0.92 \%$ variation; $\mathrm{p}<0.01)$.

\section{Conclusion}

Physicians in our study were mostly satisfied with their general health and work-life balance. Nevertheless, their mental health well-being was not satisfactory, as assessed by WEMWBS. There is a dire need for lifestyle modifications among the medical practitioners who may improve their mental and physical wellbeing subsequently allowing them to cater to their patients more effectively. It is recommended that physician-patient-specific interventions should be developed to target the health status and mental wellbeing of a physician and to encourage the physicians especially the fresh medical graduates and young doctors to indulge in healthy activities.

Categories: Public Health, Other

Keywords: lifestyle, wellbeing, physicians, wemwbs, physical activity, work life balance

\section{Introduction}

The medical profession and its training have always been so tough that it inculcates a sense of independence, competitiveness, and resilience among physicians. Almost all physicians are exposed to everyday stressors, including prolonged and irregular working hours, the threat to workplace violence, disturbed sleep cycle, emotional exhaustion, and hence, more prone to stress-related disorders and other 
diseases [1]. One in every two physicians is seen to suffer from a major health issue by the time they turn 50 [2]. However, not all physicians are simply neglecting their health; there is a pattern of barriers associated with physicians' health-seeking behavior as supported by research [1,3], with as many as $61 \%$ of physicians seen to be involved in self-diagnosis and self-treatment [1].

Over the past decade, there has been a change in this culture. Self-care has become a core competency by the Royal College of Physicians and Surgeons of Canada. Physicians are required to "demonstrate a commitment to physician health and sustainable practice [4]." Physicians are understanding that they are the main representatives of health and a healthy lifestyle. They play a leading role in advocating for a healthy lifestyle and physical activity for the masses [5]. Generally, people consider the physicians around them as role models of healthy behavior and lifestyle. Literature has shown evidence of physicians' advice in helping quit unhealthy habits such as smoking and picking up good habits [6-8].

Scientific evidence has shown that physicians who practice healthy lifestyle habits are more confident in advocating the same to their patients and even their patients are keener in adopting lifestyle modifications in an attempt to prevent chronic diseases [9]. These physicians are more likely to communicate preventive lifestyle modifications to their patients. On the other hand, physicians who do not practice healthy lifestyles are less of an advocate of preventive measures and might not discuss them with their patients [5].

Physicians' practices also determine their attitude towards advocacy of a healthy lifestyle. The physicians who take good care of their own physical and mental health counsel their patients more confidently and can relate to the barriers related to such lifestyle modifications. Physicians' attitudes towards a healthy lifestyle are important as it determines their extent of acceptance of these habits leading to overall well-being. Most people refer to their physician as the main informant regarding a healthy lifestyle [10].

Among Pakistani physicians, a discrepancy in self-practice and patient-preaching has been identified. Where they advocated a healthy lifestyle, our physicians' dietary habits, physical activity regime, and mental health were not seen to be up to the mark. A recent cross-sectional survey with more than 1000 Pakistani healthcare providers (HCPs) indicated that $76 \%$ did not exercise, $71 \%$ had $>48$ working hours per week, $>50 \%$ slept for $<7$ hours daily, and their mental health averaged to a score of 48 (maximum 70) [11]. Comparable trends of unfavorable lifestyle habits are seen among physicians in other countries such as Bahrain and Saudi Arabia [5,12,13]. Although it is very important, the emphasis on physician health has been low in Pakistan as with many other countries [14]. Physicians practicing a healthy and balanced lifestyle will also be able to advise the same to their patients. This study aimed to evaluate the lifestyle habits, wellbeing, and mental health of physicians in Pakistan.

\section{Materials And Methods}

A multi-center, cross-sectional survey was conducted across various hospitals and private clinics in Pakistan from August to October 2020. The study was approved by the institutional review board (IRB \# DSH/IRB/2020/0018). All participants were included after written informed consent.

The non-probability convenient sampling technique was adopted. The inclusion criteria consisted of physicians practicing in Pakistan as resident trainees, general practitioners (GP), and consultants, with a minimum clinical experience of three years, and those who willingly consented to participate in the specified duration of three months.

A semi-structured questionnaire was constructed which comprised of four sections. The first section consisted of socio-demographic information of the physicians including age, gender, professional status, institutional affiliation, marital status, body weight (kilograms), height (meters), chronic diseases, and family diseases. The second section consisted of lifestyle habits which included questions regarding food and drinks habits, sleep cycle, cigarette smoking, physical activity, and screen time. The third section comprised of well-being and included questions regarding self-perceived general health (participants' own view about their health as excellent, good, fair, poor), regular medical checkups, and work-life balance. The fourth section comprised mental wellbeing. It was assessed using Warwick Edinburg Mental Wellbeing Scale (WEMWBS). It is a reliable and validated tool with internal consistency and a reliability coefficient (Cronbach's alpha) of 0.87 [15]. It comprises 14-positive items and is scored on a 1-5 point Likert scale where 1 corresponds to "none of the time" and 5 corresponds to "all of the time." All questions are equally weighted. Scores can range from a minimum of 14 to a maximum of 70 points. Higher scores are associated with higher levels of mental wellbeing [15].

Data were stored and analyzed using IBM-SPSS version 23.0 (IBM Corp., Armonk, NY). Cronbach alpha was used to check the reliability of the WEMWBS scale. Mean and standard deviation (SD) were given for continuous items. Frequency and percentages were calculated for categorical items. One-way analysis of variance (ANOVA) was used to compare the mean WEMWBS scores with marital status, profession, institutional affiliation, and health in general whereas an independent sample t-test was used to compare the mean WEMWBS with respect to age group and gender. Pearson Correlation analysis was performed to study the relationship of WEMWBS scores with age, health in general, and body mass index (BMI). P-values under 0.05 were considered significant. 


\section{Cureus}

\section{Results}

There were 1,406 physicians included from various parts of the country for this survey. Most of them $(n=1284 ; 91.3 \%)$ were males and 122 (8.7\%) were females. Their mean age was $44.09 \pm 11.18$ years and their mean BMI was $25.09 \pm 5.60 \mathrm{~kg} / \mathrm{m}^{2}$. The majority $(\mathrm{n}=501 ; 43.1 \%)$ were general practitioners and the rest were consultants 418 (35.9\%) and residents 173 (14.9\%). Most of the study sample ( $\mathrm{n}=1153 ; 83.4 \%)$ were married; 188 (13.6\%) were single, and 42 (3.0\%) were separated/divorced. There were 619 (46.4\%) physicians who were practicing in public hospitals, 385 (28.5\%) were in private hospitals, and 330 (24.7\%) only practiced outpatient in the private sector. There were 664 (45.1\%), hypertensive physicians, with 731 (49.7\%) having a family history of hypertension; 520 (35.4\%) diabetic physicians with 574 (39.0\%) having a family history of diabetes; and 495 (33.7\%) hypercholesterolemic with 138 (9.4\%) having a family history of hypercholesterolemia. Most of them were prescribed angiotensin receptor blockers $(n=354 ; 24.1 \%)$ for hypertension, metformin $(n=268 ; 18.2 \%)$ for diabetes mellitus, and rosuvastatin $(n=288 ; 19.6 \%)$ for hypercholesterolemia. Only 89 (6.1\%) physicians were taking insulin for the treatment of diabetes mellitus.

There were 768 (53.0\%) physicians who reported their general health as "good." Only 206 (14.6\%) had seen an HCP for their health in the last six months while 358 (24.7\%) never went for a routine medical checkup. Around $72.8 \%(n=1045)$ of physicians were satisfied with their work-life balance and $16.4 \%(n=237)$ reported that if given the chance, they will choose another profession for themselves (Table 1).

\begin{tabular}{|l|r|}
\hline Variables & Frequency $(\%)$ \\
\hline Regular meals & \\
\hline Breakfast & $1184(78.3 \%)$ \\
Lunch & $981(64.9 \%)$ \\
Dinner & $1093(72.3 \%)$
\end{tabular}

Usual frequency of types of food

Fresh fruits

Do not eat

46 (3.0\%)

1-2 times/week

381 (25.2\%)

3-5 times/week

$703(46.5 \%)$

6-7 times/week

$321(21.2 \%)$

Fresh vegetables

Do not eat

65 (4.3\%)

1-2 times/week

437 (28.9\%)

3-5 times/week

$671(44.4 \%)$

6-7 times/week

$271(17.9 \%)$

Chicken

Do not eat

208 (13.8\%)

1-2 times/week

779 (51.5\%)

3-5 times/week

362 (23.9\%)

6-7 times/week

$72(4.8 \%)$

Fish

Do not eat

149 (9.9\%)

1-2 times/week

$864(57.1 \%)$

3-5 times/week

356 (23.5\%)

6-7 times/week

53 (3.5\%)

Red meat

Do not eat

$281(18.6 \%)$ 


\section{Cureus}

\begin{tabular}{|c|c|}
\hline 1-2 times/week & $764(50.5 \%)$ \\
\hline 3-5 times/week & $341(22.6 \%)$ \\
\hline 6-7 times/week & $41(2.7 \%)$ \\
\hline \multicolumn{2}{|l|}{ Junk food } \\
\hline Do not take & $386(26.5 \%)$ \\
\hline Daily & $96(6.6 \%)$ \\
\hline Once weekly & $321(22.0 \%)$ \\
\hline Weekly & $287(19.7 \%)$ \\
\hline Monthly & $366(25.1 \%)$ \\
\hline \multicolumn{2}{|l|}{ Frizzy drinks } \\
\hline Do not take & $456(31.5 \%)$ \\
\hline Daily & $159(11.0 \%)$ \\
\hline Once weekly & $314(21.7 \%)$ \\
\hline Weekly & $283(19.5 \%)$ \\
\hline Monthly & $237(16.4 \%)$ \\
\hline \multicolumn{2}{|l|}{ Caffeinated beverages per day } \\
\hline Do not take & $585(41.3 \%)$ \\
\hline Once & $507(35.8 \%)$ \\
\hline Twice & $234(16.5 \%)$ \\
\hline Three times or more & $92(6.5 \%)$ \\
\hline Mean glasses of water per day & $8.56 \pm 3.87$ \\
\hline \multicolumn{2}{|l|}{ Physical activity in a week } \\
\hline Yes & $800(56.3 \%)$ \\
\hline Mean minutes/day physical activity & $32.58 \pm 18.9$ \\
\hline Mean days/week physical activity & $5.92 \pm 9.80$ \\
\hline \multicolumn{2}{|l|}{ Type of physical activity } \\
\hline Lift weights & $75(6.6 \%)$ \\
\hline Run & $244(21.6 \%)$ \\
\hline Walk & $717(63.5 \%)$ \\
\hline Swim & $69(6.1 \%)$ \\
\hline Aerobics & $24(2.1 \%)$ \\
\hline Others (cricket, football, badminton, yoga, etc.) & $55(3.7 \%)$ \\
\hline \multicolumn{2}{|l|}{ Elevator or stairs use usually in multi-story buildings } \\
\hline Elevators & 209 (14.9\%) \\
\hline Stairs & $536(38.2 \%)$ \\
\hline Both & $658(46.9 \%)$ \\
\hline Mean hours of sleep per day & $7.64 \pm 1.39$ \\
\hline \multicolumn{2}{|l|}{ Smoking status } \\
\hline Current smoker & 190 (13.1\%) \\
\hline
\end{tabular}




\section{Cureus}
Ex-smoker
$220(15.2 \%)$
Never smoked
$1040(71.7 \%)$
Mean hours in daily clinical practice
Institution
$6.25 \pm 2.06$
Private
$5.30 \pm 2.73$
Mean average daily screen time
TV/DVD
$1.98 \pm 2.95$
Laptop/computer
$2.46 \pm 3.74$
Mobile
$3.75 \pm 4.66$

\section{TABLE 1: Self-reported well-being status of the physicians}

Table 2 summarizes the lifestyle habits of our sample physicians. These include their eating habits with most of the participants eating all three meals of the day. When inquired about fresh fruit intake, $46.5 \%$ $(\mathrm{n}=703)$ were found to be taking it 3-5 times/week, and for fresh vegetables, it was 3-5 times/week as well $(\mathrm{n}=671 ; 44.4 \%)$. Chicken and fish intake was $1-2$ times/week in $51.5 \%(\mathrm{n}=779)$ and $57.1 \%(\mathrm{n}=864)$, respectively. Similarly, they reported eating red meat $1-2$ times/week ( $\mathrm{n}=764 ; 50.5 \%)$ and junk food monthly $(\mathrm{n}=366 ; 25.1 \%)$ or once weekly $(321,22.0 \%)$. Most of the study participants were not used to drinking fizzy $(n=456 ; 31.5 \%)$ or caffeinated drinks $(n=585 ; 41.3 \%)$. A good proportion $(n=800 ; 56.3 \%)$ of physicians had a habit of doing physical activities at $32.58 \pm 18.9$ minutes/day and $5.92 \pm 9.80$ days/week like walking, running, and weight lifting. Their mean duration of sleep was $7.64 \pm 1.39$ hours/day while that of clinical practice was $6.25 \pm 2.06$ hours institutionally and $5.30 \pm 2.73$ in private.

\section{Regular meals}

Breakfast

$1184(78.3 \%)$

Lunch

$981(64.9 \%)$

Dinner

$1093(72.3 \%)$

Usual frequency of types of food

Fresh fruits

Do not eat

46 (3.0\%)

1-2 times/week

$381(25.2 \%)$

3-5 times/week

$703(46.5 \%)$

6-7 times/week

$321(21.2 \%)$

Fresh vegetables

Do not eat

$65(4.3 \%)$

1-2 times/week

437 (28.9\%)

3-5 times/week

$671(44.4 \%)$

6-7 times/week

271 (17.9\%)

Chicken

Do not eat

$208(13.8 \%)$

1-2 times/week

779 (51.5\%)

3-5 times/week

362 (23.9\%)

6-7 times/week

$72(4.8 \%)$ 


\section{Cureus}

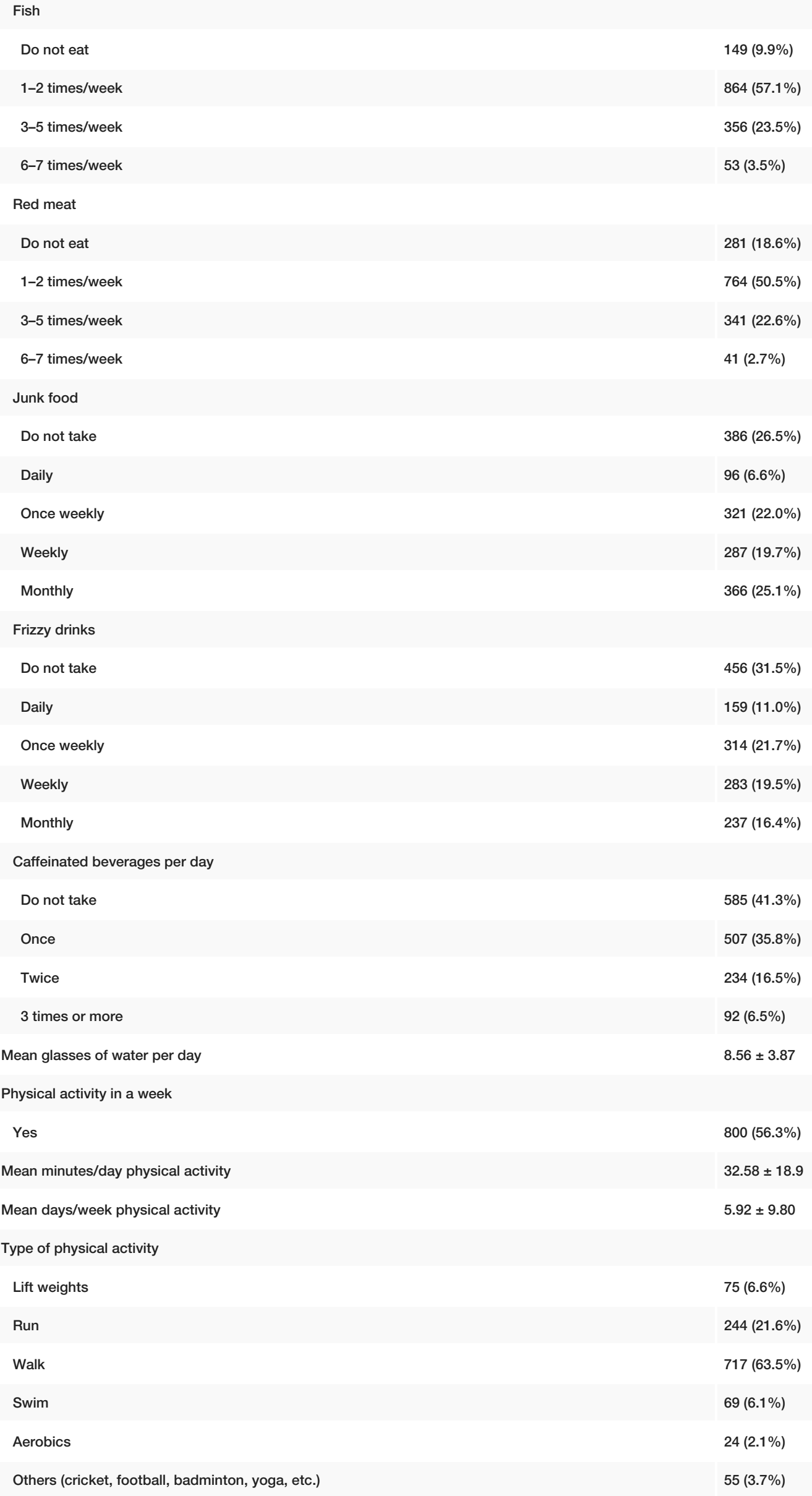

Elevator or stairs use usually in multi-story buildings 


\section{Cureus}

\begin{tabular}{|l|l|}
\hline Elevators & $209(14.9 \%)$ \\
\hline Stairs & $536(38.2 \%)$ \\
Both & $658(46.9 \%)$ \\
\hline Smoking status & $7.64 \pm 1.39$ \\
\hline Current smoker & \\
\hline Ex-smoker & $190(13.1 \%)$ \\
\hline Never smoked & $220(15.2 \%)$ \\
\hline Mean hours in daily clinical practice & $1040(71.7 \%)$ \\
\hline Institution & \\
\hline Private & $6.25 \pm 2.06$ \\
Mean average daily screen time & $5.30 \pm 2.73$ \\
\hline TV/DVD & \\
\hline Laptop/computer & $1.98 \pm 2.95$ \\
Mobile & $2.46 \pm 3.74$ \\
\hline
\end{tabular}

TABLE 2: Lifestyle habits of the physicians

Table 3 shows the descriptive statistics on WEMWBS items, where 899 samples fully completed the wellbeing questionnaire. The reliability of 14 items for the WEMWBS scale was observed at 0.87 using Cronbach alpha. The overall mean score for WEMWBS was $52.08 \pm 8.26$. The comparison of WEMWBS scores across socio-demographic factors showed that $46.3 \%$ of the sample was of $\leqslant 40$ years with a mean of $52.97 \pm 8.84$ on WEMWBS which was slightly higher than the older age group and the differences were statistically significant with $\mathrm{p}<0.01$. Male physicians also scored significantly higher on WEMWBS $(52.35 \pm 8.78)$ as compared to their female counterparts $(\mathrm{p}<0.01)$. WEMWBS scores also varied significantly across various levels of expertise - with consultants scoring the highest (52.67 \pm 9.02$)$ and others scoring the lowest (48.63 \pm $8.58 ; \mathrm{p}=0.02)$. Physicians practicing in the public hospitals only $(53.05 \pm 9.02)$, scored higher on WEMWBS as compared to those in the private hospitals $(51.28 \pm 8.12)$ as well as those practicing in private clinics only $(49.57 \pm 8.82 ; \mathrm{p}<0.01)$. Physicians who perceived their health as excellent scored highest on WEMWBS (53.55 \pm 9.31 ), than those who considered their health good (51.68 \pm 8.40 ), poor (50.71 \pm 9.27$)$, or fair $(48.70 \pm 8.15)$. The differences were statistically significant $(\mathrm{p}<0.01)$. 


\section{Cureus}

\begin{tabular}{|c|c|c|}
\hline Items & Mean & SD \\
\hline I have been feeling optimistic about the future & 3.47 & 1.164 \\
\hline I have been feeling useful & 3.69 & 1.008 \\
\hline I have been feeling relaxed & 3.68 & 0.990 \\
\hline Thave been feeling interested in other people & 3.44 & 1.150 \\
\hline Thave had the energy to spare & 3.53 & 1.054 \\
\hline I have been dealing with problems well & 3.80 & 0.993 \\
\hline I have been thinking clearly & 3.88 & 1.019 \\
\hline I have been feeling good about myself & 3.92 & 0.982 \\
\hline I have been feeling close to other people & 3.55 & 1.084 \\
\hline I have been feeling confident & 3.98 & 0.979 \\
\hline I have been able to make up my mind about things & 3.85 & 0.983 \\
\hline I have been feeling loved & 3.68 & 1.091 \\
\hline I have been interested in new things & 3.90 & 1.000 \\
\hline I have been feeling cheerful & 3.73 & 1.022 \\
\hline Reliability coefficient Cronbach's a & 14-items & 0.87 \\
\hline
\end{tabular}

TABLE 3: Descriptive statistics on WEMWBS items $(n=899)$

The correlation analysis for WEBWBS scores with age, health in general, and BMI showed a significant negative correlation of WEMWBS scores with health in general and age. A 2.5\% variation in WEMWBS explained by health in general and a $0.92 \%$ variation by age. These were considered statistically significant $(\mathrm{p}<0.01)$. However, there was no significant correlation observed between WEMWBS and BMI.

\section{Discussion}

Stressful environments such as those of a patient-loaded hospital can lead to delayed or even misdiagnosis of diseases. Furthermore, due to long and exhausting work hours, physicians tend to overlook their own symptoms, hence, do not get a professional assessment for their conditions. Similarly, due to exhausting work hours, many physicians do not indulge in physical exercise or stick to their dietary regimes. They do not find the physical or mental strength to take care of themselves after working endless hours each day. This directly affects the quality of healthcare they provide to their patients $[16,17]$. The current study explored the lifestyle habits, wellbeing, and mental health of Pakistani physicians. This can be a step forward to develop certain national-level policies for improving the work efficiency of these HCPs.

The majority of the participants were satisfied with their health status; however, they scored poorly when it came to their mental health. Moreover, gender discrepancy was prominently noticed in this mental wellbeing assessment by the WEMWBS with males scoring significantly higher $(\mathrm{p}<0.01)$. This inequality of male to female physician ratio has been observed, globally [18]. Our findings were in accordance with a 2017 study by Khattak et al., where a physician's physical and mental health were assessed, concluding that female physicians struggled with maintaining healthy activities affecting their overall health and well-being [19]. The authors recommend that to ensure physicians' well-being, novice interventional programs could be arranged that target this neglected area.

Duty hours of doctors and the need to restrict them have been long debated in the medical literature. For instance, a study from Indonesia, exploring the prevalence and risk factors of gastroesophageal reflux disease (GERD) in medical practitioners reported long working hours to be a contributing factor with doctors in Indonesia working 51-67 hours per week on average. They are under extremely stressful circumstances and the majority of the doctors are under constant worry about their patients [20]. Countries like the United States and New Zealand have managed to restrict doctor duty hours to 16-24 hours; however, in Pakistan, the situation is still unregulated [17]. Pakistani doctors are still working for as many as 74-92 hours weekly and are shown to exhibit high stress and low job satisfaction [21]. In the current study, the majority of the participants claimed that the most physical activity they get is by walking which includes walking from one department to the other. Only about one-fourth population confessed that they indulged in smoking which 
could be an understatement as many young residents or physicians tend to hide their habit of smoking. These unhealthy activities lead to poor health status among physicians $[20,22,23]$.

One striking finding in our study was how one-fourth of the participants never visited a general health practitioner for a routine health examination. Further review of the literature divulged similar findings. In 2012, a review was conducted on preventive healthcare and lifestyle behaviors of medical practitioners towards their health [23]. It was concluded that there is a culture that prevents doctors from seeing other healthcare professionals when they feel sick. Doctors are often portrayed as heroes who do not need to seek any medical attention and their sole purpose is to serve patients. Asking for help is looked upon as weakness and those who do ask for help are judged. However, physicians who are physically and mentally fit and consider their health as a priority tend to provide better healthcare to their patients [22,23].

Wiskar in a review discussed the option of counseling physicians who indulge in detrimental habits and ignore their health, both mental and physical to fulfill their obligations as a physician [23]. Many doctors avoid going to a professional healthcare practitioner even when they are fully aware of their symptoms because there is a widely popular culture of associating a physician's own mental and physical health with his/her medical competence. This is why many physicians tend to hide their conditions to avoid being judged by their fellow practitioners [22].

Not only does the health of a physician directly impact the delivery of the healthcare services to their patients but also affects the way they counsel their patients. In a Canadian study, it was found that physicians who were non-smokers were able to counsel their patients about smoking hazards actively and more confidently [24].

A study in 2014 proposed the implementation of a revised system in the medical field to allow the physician to take care of themselves [25]. The authors focus on work-life balance for not only physicians but also for other personnel of the hospital staff since these people are prone to suffer from work-related burnout and mental exhaustion. The study highlighted that it is important for the physician to be physically and mentally fit to provide better healthcare to their patients. The significance of work-life balance among healthcare professionals is highlighted in the current study as over one-fourth of the physicians were either not satisfied with their work-life balance or were unsure about it.

Another study reported that around $46 \%$ of doctors in the United States have felt frustrated with the system at some point in their careers [26]. In another study, $73 \%$ of the internists and $68 \%$ of the physicians claimed that they would reconsider their field of the profession if it was an option [27]. Contrary to that, our findings reported that only about one-sixth of the physicians wished to change their fields. Many of these physicians suffered from work-related burnout and reported a lack of interest in the activities of daily life, indulged in substance abuse, and had suicidal thoughts. These issues are preventable with certain modifications such as reasonable work hours, increased salary, increased leisure time, and indulging in self-care activities.

A study was conducted on the lifestyle of physicians highlighting the consumption of an unbalanced diet by the physicians [23]. Most of the physicians admitted to not having proper meals or a stable sleeping schedule due to their demanding jobs. The physicians were badly affected due to poor nutrition which subsequently affected their behavior and decision-making capabilities. The majority of the participants in our study consumed junk food (such as sweets, sweetened beverages, fast foods, and salty snacks) and carbonated drinks. Poor diet is associated with high-stress levels and poor cognitive functions [28].

Despite the many apparent strengths of the current study, there were some limitations. First, the participants were selected using the non-probability convenience sampling technique which may have raised the selection bias hence, limiting the interpretation of the findings. Second, the majority of the participants were male and married. Therefore, it impacts the generalizability of the data and limits the implication of the findings to the entire country.

\section{Conclusions}

Physicians in our study were mostly satisfied with their general health and work-life balance. Nevertheless, their mental health well-being was not satisfactory, as assessed by WEMWBS. There is a dire need for lifestyle modifications among the medical practitioners who may improve their mental and physical wellbeing subsequently allowing them to cater to their patients more effectively. It is recommended that physician-patient-specific interventions should be developed to target the health status and mental wellbeing of a physician and to encourage the physicians especially the fresh medical graduates and young doctors to indulge in healthy activities.

\section{Additional Information \\ Disclosures}

Human subjects: Consent was obtained or waived by all participants in this study. Institutional Review Board issued approval IRB \# DSH/IRB/2020/0018. Animal subjects: All authors have confirmed that this 
study did not involve animal subjects or tissue. Conflicts of interest: In compliance with the ICMJE uniform disclosure form, all authors declare the following: Payment/services info: All authors have declared that no financial support was received from any organization for the submitted work. Financial relationships: All authors have declared that they have no financial relationships at present or within the previous three years with any organizations that might have an interest in the submitted work. Other relationships: All authors have declared that there are no other relationships or activities that could appear to have influenced the submitted work.

\section{Acknowledgements}

We are thankful to Clinision the research domain of PharmEvo Pvt. Ltd. for providing administrative support for this research.

\section{References}

1. Benkhadra K, Adusumalli J, Rajjo T, Hagen PT, Wang Z, Murad MH: A survey of health care needs of physicians. BMC Health Serv Res. 2016, 16:472. 10.1186/s12913-016-1728-4

2. Shanafelt TD, Noseworthy JH: Executive leadership and physician well-being: nine organizational strategies to promote engagement and reduce burnout. Mayo Clin Proc. 2017, 92:129-46. 10.1016/j.mayocp.2016.10.004

3. George S, Hanson J, Jackson JL: Physician, heal thyself: a qualitative study of physician health behaviors . Acad Psychiatry. 2014, 38:19-25. 10.1007/s40596-013-0014-6

4. The CanMEDS 2005 physician competency framework . (2005). Accessed: May 05, 2021: http://www.ub.edu/medicina_unitateducaciomedica/documentos/CanMeds.pdf.

5. Borgan SM, Jassim GA, Marhoon ZA, Ibrahim MH: The lifestyle habits and wellbeing of physicians in Bahrain: a cross-sectional study. BMC Public Health. 2015, 15:655. 10.1186/s12889-015-1969-X

6. Borgan SM, Marhoon ZA, Whitford DL: Beliefs and perceptions toward quitting waterpipe smoking among cafe waterpipe tobacco smokers in Bahrain. Nicotine Tob Res. 2013, 15:1816-21. 10.1093/ntr/ntt064

7. Rose SA, Poynter PS, Anderson JW, Noar SM, Conigliaro J: Physician weight loss advice and patient weight loss behavior change: a literature review and meta-analysis of survey data. Int J Obes (Lond). 2013, 37:11828. 10.1038/ijo.2012.24

8. Stead LF, Buitrago D, Preciado N, Sanchez G, Hartmann-Boyce J, Lancaster T: Physician advice for smoking cessation. Cochrane Database Syst Rev. 2013, CD000165. 10.1002/14651858.CD000165.pub4

9. Oberg EB, Frank E: Physicians' health practices strongly influence patient health practices. J R Coll Physicians Edinb. 2009, 39:290-1. 10.4997/JRCPE.2009.422

10. Abramson S, Stein J, Schaufele M, Frates E, Rogan S: Personal exercise habits and counseling practices of primary care physicians: a national survey. Clin J Sport Med. 2000, 10:40-8. 10.1097/00042752-20000100000008

11. Ahmad W, Taggart F, Shafique MS, et al.: Diet, exercise and mental-wellbeing of healthcare professionals (doctors, dentists and nurses) in Pakistan. PeerJ. 2015, 3:e1250. 10.7717/peerj.1250

12. Alshareef SJ, Alzahrani A, Farahat FM: Lifestyle habits and well-being among primary health physicians in western Saudi Arabia. J Public Health. 2019, 27:57-62. 10.1007/s10389-018-0929-1

13. Alsuwaida A, AlSharidi A, AlAnazi N, et al.: Health and well-being among physicians. Intern Med J. 2013, 43:1310-5. 10.1111/imj.12249

14. Atif K, Khan HU, Ullah MZ, Shah FS, Latif A: Prevalence of anxiety and depression among doctors; the unscreened and undiagnosed clientele in Lahore, Pakistan. Pak J Med Sci. 2016, 32:294-8. 10.12669/pjms.322.8731

15. Cilar L, Pajnkihar M, Štiglic G: Validation of the Warwick-Edinburgh Mental Well-Being scale among nursing students in Slovenia. J Nurs Manag. 2020, 28:1335-46. 10.1111/jonm.13087

16. Goldberg DM, Cho BY, Lin HC: Factors influencing U.S. physicians' decision to provide behavioral counseling. Prev Med. 2019, 119:70-6. 10.1016/j.ypmed.2018.12.015

17. Sameer-ur-Rehman, Kumar R, Siddiqui N, Shahid Z, Syed S, Kadir M: Stress, job satisfaction and work hours in medical and surgical residency programmes in private sector teaching hospitals of Karachi, Pakistan. J Pak Med Assoc. 2012, 62:1109-12.

18. Henderson JT, Weisman CS: Physician gender effects on preventive screening and counseling: an analysis of male and female patients' health care experiences. Med Care. 2001, 39:1281-92. 10.1097/00005650200112000-00004

19. Khattak AF, Mahsood N, Jan AS, Wahid I: Physicians' physical and mental health status and their personal health practices in tertiary care hospitals. Int J Pathol. 2017, 15:171-176.

20. Syam AF, Hapsari PF, Makmun D: The prevalence and risk factors of GERD among Indonesian medical doctors. Makara J Health Res. 2016, 20:35-40. 10.7454/msk.v20i2.5740

21. Juma AA, Hosein S: Resident duty hours: globally accepted standards and the situation in Pakistan . J Pak Med Assoc. 2011, 61:1253.

22. Thompson WT, Cupples ME, Sibbett CH, Skan DI, Bradley T: Challenge of culture, conscience, and contract to general practitioners' care of their own health: qualitative study. BMJ. 2001, 323:728-31. 10.1136/bmi.323.7315.728

23. Wiskar K: Physician health: a review of lifestyle behaviors and preventive health care among physicians . BC Med J. 2012, 54:419-23.

24. Frank E, Segura C, Shen H, Oberg E: Predictors of Canadian physicians' prevention counseling practices . Can J Public Health. 2010, 101:390-5. 10.1007/BF03404859

25. Bodenheimer T, Sinsky C: From triple to quadruple aim: care of the patient requires care of the provider . Ann Fam Med. 2014, 12:573-6. 10.1370/afm.1713

26. Berwick DM: What 'patient-centered' should mean: confessions of an extremist. Health Aff (Millwood). 


\section{Cureus}

2009, 28:w555-65. 10.1377/hlthaff.28.4.w555

27. Medscape physician compensation report 2014. (2014). Accessed: May 05, 2021:

https://www.medscape.com/features/slideshow/compensation/2014/public/overview.

28. Gómez-Pinilla F: Brain foods: the effects of nutrients on brain function . Nat Rev Neurosci. 2008, 9:568-78. 10.1038/nrn2421 